\title{
Laparoscopic and Open Surgical Nephrectomy for Xanthogranulomatous Pyelonephritis
}

\author{
HERKANWAL S. KHAIRA, M.D., ${ }^{1}$ RAJAL B. SHAH, M.D., ${ }^{1,2}$ and J. STUART WOLF, JR., M.D. ${ }^{1}$
}

\begin{abstract}
Background and Purpose: Xanthogranulomatous pyelonephritis (XGP) is a severe, chronic renal-parenchymal infection. Nephrectomy is the treatment of choice. Because of the renal and perirenal inflammatory changes that commonly accompany XGP, the laparoscopic approach is difficult. We compared our experience with laparoscopic and open surgical nephrectomy for XGP.

Patients and Methods: A retrospective chart review of all adult nephrectomy specimens with the pathologic diagnosis of XGP between January 1997 and May 2003 was performed. Preoperative presentation, operative details, and postoperative recovery and complications were included in the data collection.

Results: Three patients approached laparoscopically and eight patients approached with open surgery were found to have XGP on pathologic analysis. The disease was suspected preoperatively in all patients. Among the laparoscopically treated patients, there was $1(33 \%)$ who suffered major complications; this was the only patient who required conversion to open surgery. Among the open-surgical group, there were 2 (22\%) major and $3(33 \%)$ minor complications. Postoperative hospitalization was longer in the open-surgical group (mean $13.7 v 4.7$ days), and when the case of open conversion was excluded, narcotic use was less in the laparoscopy group.

Conclusions: The treatment of some XGP cases with laparoscopic nephrectomy is a possible, albeit challenging, option. The incidences of intraoperative and postoperative complications were roughly equivalent in the laparoscopic and open-surgery patients in our study. If completed, laparoscopy appears to be associated with decreased postoperative morbidity. However, this may represent selection bias, and larger, prospective studies may better define the suspected benefit.
\end{abstract}

\section{INTRODUCTION}

$\mathbf{X}^{\prime}$ ANTHOGRANULOMATOUS PYELONEPHRITIS (XGP), first described in 1916 by Schlagenhaufer, ${ }^{1}$ is a form of severe, chronic bacterial infection of the renal parenchyma. The disease is commonly associated with renal lithiasis with uppertract obstruction. Hydronephrosis and severely diminshed renal function are typical features. ${ }^{2,3}$ Histologically, XGP is marked by the replacement of normal renal parenchyma with lipidladen, foamy macrophages (xanthoma cells). The disease more commonly affects middle-aged women and usually presents unilaterally, with fever, flank pain, persistent bacteruria, or some combination. ${ }^{4}$ Misdiagnosis of XGP is not uncommon; the condition has been called the "great imitator" because of similarities to other inflammatory and neoplastic conditions that affect the kidney. ${ }^{2,5}$ The etiology of the disease, although not fully elucidated, is associated with obstructive uropathy and infection with either Proteus mirabilis or Escherichia coli.

The prepathologic diagnosis of XGP can be reasonably made with CT when certain consistent findings are present (Fig. 1). ${ }^{6}$ Specifically, low-attenuation lesions, between 10 and $15 \mathrm{HU}$, with little or no contrast uptake, will likely be noted. ${ }^{7}$ Not uncommonly, these low-attenuation regions will border significant collecting-system dilation or normal, but compressed, functioning renal parenchyma. The extent of XGP can be well determined with CT scanning; involvement in the retroperitoneum, contiguous organs, and extension into Gerota's fascia can all be defined. ${ }^{8,9}$

The treatment of choice for XGP is nephrectomy, although obliterated tissue planes and inflammatory involvement of the renal hilum and contiguous structures often make this a challenging procedure. Because of the difficulties commonly asso-

Departments of ${ }^{1}$ Urology and ${ }^{2}$ Pathology, University of Michigan, Ann Arbor, Michigan. 


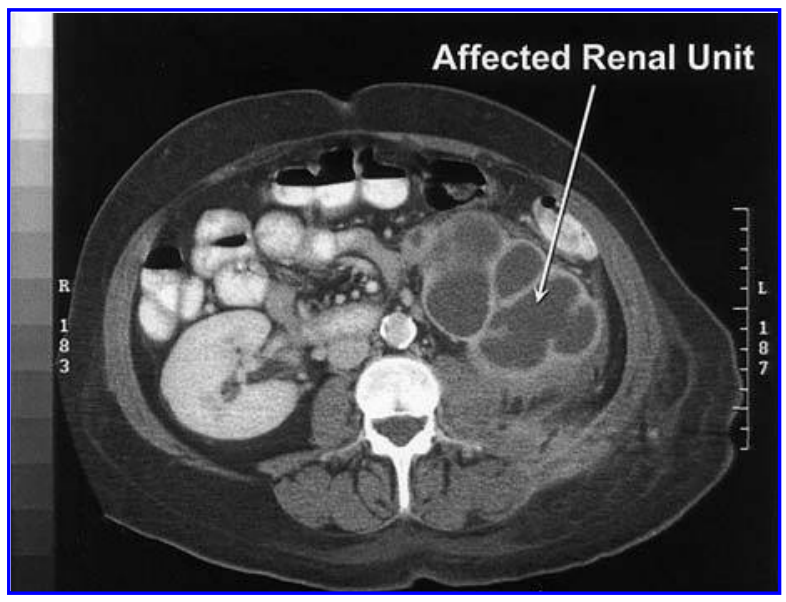

FIG. 1. Contrast-enhanced CT image showing hydronephrotic kidney with xanthogranulomatous pyelonephritis.

ciated with XGP nephrectomy, few have reported laparoscopic nephrectomy in this situation. Laparoscopic nephrectomy has been used for treatment of some XGP cases at our institution in recent years. We evaluated our experience with laparoscopic and open nephrectomy for XGP kidneys during the past 7 years to determine if either procedure provided an advantage to patient care.

\section{PATIENTS AND METHODS}

\section{Patients}

After obtaining Institutional Review Board consent, a review was conducted of all adult nephrectomy specimens at the University of Michigan Hospitals between January 1997 and May 2003 that revealed 12 specimens with the pathologic diagnosis of XGP. A small focus of XGP was found in a small atrophic nephrectomy specimen during attempted bilateral aortorenal revascularization, and this subject was excluded from analysis. Of the remaining 11 subjects, 3 were initially treated with laparoscopic nephrectomy and 8 similar patients with open surgery (Table 1). The laparoscopic approaches were handassisted transperitoneal $(\mathrm{N}=2)$ and standard retroperitoneoscopic $(\mathrm{N}=1)$. All eight of the open procedures were performed through a flank approach.
The medical records for the 11 patients were reviewed, taking into account the details of demographics, medical history, presenting characteristics, operative details, and postoperative outcomes, including complications. Laparoscopic nephrectomy was considered an option on the basis of the radiographic imaging of the affected kidney. Inflammatory renal units that radiographically appeared to be contained within Gerota's fascia were considered amenable to laparoscopic nephrectomy. It was felt that those renal units could be removed outside Gerota's fascia, thereby reducing the risk of infection dissemination during transperitoneal surgery. A hand-assisted transperitoneal approach was preferred because it offered greater control of the operative field. A retroperitoneoscopic approach was selected in one patient beause the inflammatory process appeared radiographically contained within the kidney, and the patient had had extensive prior abdominal surgery. Prior to treatment, patients who were offered the laparoscopic approach as a treatment option were informed that the utility of laparoscopic nephrectomy had not yet been established for the treatment of XGP and that conversion to open surgery was a possibility.

\section{Laparoscopic surgical technique}

The retroperitoneoscopic approach utilized the four-port distribution and technique previously described by Gill. ${ }^{10}$ The specimen was removed after morcellation in a laparoscopic bag. The hand-assisted laparoscopic nephrectomies were performed through an 8-cm midline periumbilical incision with two 12$\mathrm{mm}$ ports, one at the level of the umbilicus/midclavicular line and one $3 \mathrm{~cm}$ below the costal margin at the anterior axillary line. A $5-\mathrm{mm}$ port was also placed $3 \mathrm{~cm}$ above the iliac spine at the anterior axillary line and typically was used for retraction. The colon was initially reflected medially, followed by superior detachment of the liver or spleen. Oftentimes, dense fibrotic adhesions were encountered, and nearly all dissection was performed sharply with hook electrocautery.

The renal-hilar dissection was typically challenging. In the cases that were completed by a laparoscopic approach, the vessels were taken separately with the endoscopic stapler and clips.

In the hand-assisted laproscopic nephrectomy, the specimen was removed intact through the midline incision. The renal unit was mobilized outside Gerota's fascia in order to prevent entering renal abscesses. However, if there was any gross purulence spillage during the procedure, a closed bulb-suction drainage system was left in place. The drain was removed when output was negligible, and the Foley catheter was typically removed on postoperative day 1 .

Table 1. Patient Characteristics

\begin{tabular}{lccr}
\hline & $\begin{array}{c}\text { Open surgery } \\
(\mathrm{N}=8)\end{array}$ & $\begin{array}{c}\text { Laparoscopy } \\
(\mathrm{N}=3)\end{array}$ & Total \\
\hline Mean age (years) (range) & $54.4(29-83)$ & $42.5(36-57)$ & 51.2 \\
Nephrolithiasis (\%) & $5(63)$ & $3(100)$ & $8(73)$ \\
Culture-positive infections $\quad$ (including urine, renal abscess) (\%) & $6(75)$ & $2(67)$ & $8(73)$ \\
Flank pain (\%) & $2(25)$ & $1(33)$ & $3(27)$ \\
Elevated serum creatinine (\%) & $7(88)$ & $3(100)$ & $8(73)$
\end{tabular}




\section{RESULTS}

Three patients were initially approached with laparoscopic nephrectomy, with one conversion to open surgery, and eight patients were managed with open nephrectomy. The option of laparoscopic versus open surgical nephrectomy was offered to three of the four patients who consulted a urologist with laparoscopic experience, and all three requested and underwent the procedure. The one patient who consulted a urologist with laparoscopic experience but was treated with open nephrectomy had a concurrent enterocutaneous fistula; the open nephrectomy was performed at the same time as partial colectomy and endcolostomy in order to spare the patient separate operations. Of the remaining patients who underwent open surgical nephrectomy, none was offered the option of laparoscopic nephrectomy. This may be in part because those patients consulted urologists who do not normally perform laparoscopic nephrectomy, the disease process appeared too advanced for treatment laparoscopically, or the surgeon considered laparoscopic nephrectomy untenable for the treatment of suspected XGP.

All three of the laparoscopically treated patients presented with a history of renal dysfunction, pyelonephritis, recurrent renal calculi, or some combination. An inflammatory mass was seen radiographically, and XGP was considered in the differential diagnosis in all of these patients. All eight of the patients treated with open nephrectomy had similar presentations, with radiographic evidence of a renal mass, a history of renal disease, or both (see Table 1).

The estimated blood loss (EBL) for the laparoscopy group was $1117 \mathrm{~mL}$; excluding the patient converted to open surgery, the average was $175 \mathrm{~mL}$. The average EBL for the open-surgery group was $911 \mathrm{~mL}$. The operative time for laparoscopy was longer by $52 \%$. The average length of hospital stay (LOS) for the laparoscopy group was 4.7 days, including the patient converted to open surgery. The mean LOS for the open-surgery group was much greater, at 13.7 days. Final pathologic analysis confirmed the diagnosis of XGP in all patients. Outcome data for the two groups are summarized in Table 2.

In the laparoscopy group, one patient experienced complications related to treatment. This procedure was converted to open surgery because of a renal-vein injury that could not be controlled laparoscopically. The initial approach for this patient was hand-assisted laparoscopy. The $8-\mathrm{cm}$ midline incision for the hand-assistance device was extended, and the open completion of the case was uneventful. The patient did require transfusion of 2 units of packed red blood cells postoperatively. Following discharge on postoperative day 9 , this patient (with a T1-level spinal-cord injury and a history of bowel dysfunction) was readmitted with ileus that necessitated parental nutrition and bowel rest.

In the open-surgery group, five of the eight patients experienced a postoperative complication; three were minor and two were major. One patient suffered an unrecognized bowel injury that necessitated reexploration and ileostomy on postoperative day 2. Another patient was found to have pneumothorax postoperatively that was managed with tube thoracotomy. Three patients had minor complications, either postoperative anemia necessitating transfusion $(\mathrm{N}=2)$ or postoperative pneumonia $(\mathrm{N}=1)$.

Average parental postoperative opioid use was higher in the group initially treated with laparoscopic nephrectomy. Excluding the conversion to open surgery, however, 24-hour and total parental analgesia requirements trended lower in the laparoscopy group $(33.0 \vee 36.9 \mathrm{mg}$ and $35.0 \vee 85.5 \mathrm{mg}$, respectively).

\section{DISCUSSION}

Laparoscopic nephrectomy is performed routinely for organ donation and oncologic resection, with demonstrated benefits that include diminished analgesic requirements and shorter postoperative convalescence compared with open nephrectomy. ${ }^{11,12}$ Our study sought to determine if the advantages of laparoscopic nephrectomy were applicable to the surgical treatment of XGP. This retrospective review covered 11 patients who were found to have a pathologically confirmed diagnosis of XGP after either open surgical or laparoscopic nephrectomy.

The only other case series that included both laparoscopic and open nephrectomy consisted of five laparoscopic and four open nephrectomies for XGP. ${ }^{13}$ In that series, among the laparoscopically treated patients, there was one conversion to

Table 2. Operative and Postoperative Data

\begin{tabular}{|c|c|c|c|}
\hline & $\begin{array}{l}\text { Open surgery } \\
\quad(\mathrm{N}=8)\end{array}$ & $\begin{array}{c}\text { Intended } \\
\text { laparoscopy } \\
(\mathrm{N}=3)\end{array}$ & $\begin{array}{c}\text { Completed } \\
\text { laparoscopy } \\
(\mathrm{N}=2)\end{array}$ \\
\hline Mean OR time (min) & 183 & 279 & 257 \\
\hline Mean ASA score & 2.8 & 2.5 & 2.0 \\
\hline $\begin{array}{l}\text { Mean parental analgesic requirements } \\
\text { during first } 24 \text { hours }^{\mathrm{a}}\end{array}$ & 36.9 & 46.9 & 33.0 \\
\hline Mean total parental analgesic requirements $\mathrm{s}^{\mathrm{a}}$ & 85.5 & 258.7 & 35.0 \\
\hline Mean LOS after operation (days) & $13.7^{\mathrm{b}}$ & 4.7 & 2.5 \\
\hline Mean time to first regular oral intake (days) & 2.6 & 3.7 & 1.5 \\
\hline Mean EBL (mL) & 911 & 1117 & 175 \\
\hline Major complications, \% & 25 & 33 & 0 \\
\hline Minor complications, \% & 38 & 0 & 0 \\
\hline
\end{tabular}

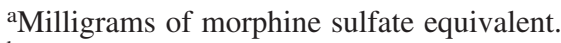

${ }^{b}$ Excluding postoperative stay to start inpatient chemotherapy. 
open surgery. Additionally, the average laparoscopic operative time was greater than double that of the open group. Narcotic pain medication use and hospital stay did not differ between the groups. The EBL averaged $260 \mathrm{~mL}$ for the laparoscopically treated group and $438 \mathrm{~mL}$ for the open-surgery group. The authors of that study concluded that laparoscopic nephrectomy for XGP did not appear to provide benefit over open surgery.

We report on three laparoscopic nephrectomies and eight open-surgical operations for XGP. We found that the LOS was shorter for the laparoscopy patients, even when there was conversion to open surgery. Although average narcotic use was greater among patients in whom laparoscopy was attempted, it was less than that associated with open surgery if the procedure could be completed laparoscopically. The average age of the patients in two groups was not dissimilar, and the clinical presentations were similar (see Table 1). Statistical analysis was not appropriate, given that there were only three subjects in one group.

Despite the challenges posed by operating on the XGP kidney laparoscopically, the surgeon was able to complete two of the three cases without untoward event or complication. In the laparoscopic case that required conversion to open surgery, there were additional complications of blood-loss anemia necessitating transfusion and recurrent ileus leading to readmission. Among the open-surgery group, there were two major and three minor complications. This similar complication rate suggests that the laparoscopic approach does not pose a greater risk when performed on properly selected cases of XGP by an experienced laparoscopic surgeon. Furthermore, there were some benefits of laparoscopy that we suspected; particularly, a trend was noted toward a shorter hospital stay and earlier recovery. However, this study is a retrospective review of a small, nonrandomized population. A bias may have existed in selecting subjects for the laparoscopic approach, which may have been related to referral patterns. The laparoscopic nephrectomytreated renal units were all contained within Gerota's fascia, and although no laparoscopy-trained surgeon demurred from treating the suspected cases of XGP through a minimally invasive approach, more advanced cases were not encountered.

Although the subject is debated, most investigators accept that obstructive uropathy and bacterial infection are key etiologic factors in XGP. ${ }^{14}$ The obstruction is often related to staghorn calculi or congenital defects. Interestingly, 1 of the 11 patients included in our study did not have these characteristics but did have transitional-cell carcinoma of the bladder, with extensive muscle-invasive disease that resulted in ureteral obstruction. Despite the concomitant malignant disease, XGP was suspected preoperatively when imaging demonstrated pyonephrosis and poor renal function.

Classically, a renal mass in the presence of urinary-tract infection, poor renal function, and renal calculi is highly suggestive of XGP. ${ }^{15}$ However, preoperative diagnosis of XGP can be challenging. Patients may not present with the classic tetrad described above. Instead, fever, flank pain, abdominal mass, bacteriuria, and other symptoms of various intensities, may occur incidentally or not at all. ${ }^{16,17}$ Nevertheless, all of pathologically confirmed diagnoses of XGP in this study population were suspected preoperatively.

The laparoscopic surgeon in our study was well experienced in minimally invasive surgery: the laparoscopic nephrectomies in this series represented the surgeon's $34^{\text {th }}, 398^{\text {th }}$, and $518^{\text {th }}$ laparoscopic procedure. The open surgical conversion was the most recent case. As a result, the applicability of our findings should be considered with prudence. For the novice laparoscopist, laparoscopic nephrectomy of an XGP kidney is not recommended. However, experienced laparoscopists should not consider XGP an absolute deterrent to performing a nephrectomy through a laparoscopic approach. Both transperitoneal hand assistance and retroperitoneal approaches were used successfully for the laparoscopic nephrectomies in this series. Generally, we prefer the transperitoneal hand-assisted approach for removal of large inflammatory masses. However, the retroperitoneoscopic approach is preferred when there is a small renal unit and extensive prior intra-abdominal surgery. Hand-assisted laparoscopy offers the added facility of an intraperitoneal hand for operative management, as well as an incision allowing intact specimen removal. Our one conversion to open surgery occurred in a hand-assisted laparoscopic operation. During that case, a renal-vascular injury occurred during dissection of the hilum. The intraperitoneal hand provided local compression while repair was attempted. However, because of the challenges related to dissection and exposure of the fibrotic XGP kidney, hemostasis could not be attained. After significant blood loss, laparoscopic repair of the injury was forfeited; the hand-assistance incision was extended to convert the procedure to open surgery. The patient's postoperative course was notable for prolonged ileus, and it could be argued that early conversion would have been beneficial after the initial vascular injury.

\section{CONCLUSION}

For many conditions, laparoscopic nephrectomy offers significant benefits over traditional open nephrectomy. These benefits have not yet been demonstrated for laparoscopic nephrectomy of the XGP kidney. In our case series, we reviewed a single institution's 7-year history of surgical treatment of XGP. We identified, among patients for whom nephrectomy was completed laparoscopically, a trend toward faster postoperative convalenscence compared with open nephrectomy and equivalent complication rates. We suggest that experienced laparoscopic surgeons consider laparoscopic management of selected cases of XGP in order to further define which of these patients may benefit from laparoscopic nephrectomy.

\section{REFERENCES}

1. Schlagenhaufer F. Uber eigentumliche Staphylomykosen der Nieren und des pararenalen Bindegewebes. Frankfurt Z Path. 1916;19:139.

2. Malek RS, Elder JS. Xanthogranulomatous pyelonephritis: A Critical analysis of 26 cases and of the literature. J Urol 1978;119:589.

3. Perez LM, Thrasher JB, Anderson EE. Successful management of bilateral xanthogranulomatous pyelonephritis by bilateral partial nephrectomy. J Urol 1993;149:100.

4. Chuang CK, Lai MK, Chang PL, et al. Xanthogranulomatous pyelonephritis: Experience in 36 cases. J Urol 1992;147:333.

5. Anhalt MA, Cawood CD, Scott R Jr. Xanthogranulomatous pyelonephritis: A comprehensive review with report of 4 additional cases. J Urol 1971;105:10. 
6. Dembry LM, Andriole VT. Renal and perirenal abscesses. Infect. Dis Clin North Am 1997;11:663.

7. Subramanyam BR, Megibow AJ, Raghavendra BN, et al. Diffuse xanthogranulomatous pyelonephritis: Analysis by computed tomography and sonography. Urol Radiol 1982;4:5.

8. Eastham J, Ahlering T, Skinner E. Xanthogranulomatous pyelonephritis: Clinical findings and surgical considerations. Urology 1994;43:295.

9. Kaplan DM, Rosenfeld AT, Smith RC. Advances in the imaging of renal infection: Helical CT and modern coordinated imaging. Infect Dis Clin North Am 1997;11:681.

10. Gill IS. Retroperitoneal laparoscopic nephrectomy. Urol Clin North Am 1998;25:343.

11. Allan JD, Tolley DA, Kaouk JH, et al. Laparoscopic radical nephrectomy. Eur Urol 2001;40:17.

12. Ratner LE, Ciseck LJ, Moore RG, et al. Laparoscopic live donor nephrectomy. Transplantation 1995;60:1047.

13. Bercowsky E, Shalhav AL, Portis A, et al. Is the laparoscopic approach justified in patients with xanthogranulomatous pyelonephritis? Urology 1999;54:437.
14. Rosi P, Selli C, Carini M, et al. Xanthogranulomatous pyelonephritis: Clinical experience with 62 cases. Eur Urol 1986;12:96.

15. Goodman M, Curry T, Russell T. Xanthogranulomatous pyelonephritis (XGP): A local disease with systemic manifestations: Report of 23 patients and review of the literature. Medicine 1979;58:171.

16. Figueroa AJ, Stein JP, Cunningham JA, et al. Xanthogranulomatous pyelonephritis in a pregnant woman: A case report and review of the literature. Urology 1996;48:294.

17. Tolia BM, Iloreta A, Freed SZ, et al. Xanthogranulomatous pyelonephritis: Detailed analysis of 29 cases and a brief discussion of atypical presentations. J Urol 1981;126:437.

Address reprint requests to: J. Stuart Wolf, M.D.

Dept. of Urology, Box 0330

University of Michigan

1500 E. Medical Center Drive

Ann Arbor, MI 48109-0330

E-mail: Wolfs@med.umich.edu 


\section{This article has been cited by:}

1. Thomas J. Guzzo, Trinity J. Bivalacqua, Phillip M. Pierorazio, John Varkarakis, Edward M. Schaeffer, Mohamad E. Allaf. 2009. Xanthogranulomatous pyelonephritis: presentation and management in the era of laparoscopy. BJU International . [CrossRef]

2. Ricardo J. Duarte, Anuar I. Mitre , José L. Chambô , Marco A. Arap, Miguel Srougi . 2008. Laparoscopic Nephrectomy Outside Gerota Fascia for Management of Inflammatory KidneyLaparoscopic Nephrectomy Outside Gerota Fascia for Management of Inflammatory Kidney. Journal of Endourology 22:4, 681-686. [Abstract] [PDF] [PDF Plus]

3. Hyun Kee Cho, Doo Sang Kim, Dong Soo Ryu, Tae Hee Oh, Youn Soo Jeon. 2008. Retroperitoneal Laparoscopic Nephrectomy for Inflammatory Renal Diseases. Korean Journal of Urology 49:2, 107. [CrossRef]

4. Lutfi Tunc, Hasan Biri, Metin Onaran, Mustafa Krac, Süleyman Yesil, Ibrahim Bozkirli. 2008. Laparoscopic Nephrectomy for Xanthogranulomatous Pyelonephritis in the Absence of Kidney Stones or Clinical Urinary Infection. Surgical Laparoscopy, Endoscopy \& Percutaneous Techniques 17:6, 570-572. [CrossRef]

5. Joseph C. Liao, Alberto Breda, Peter G. Schulam. 2007. Laparoscopic renal surgery for benign disease. Current Urology Reports 8:1, 12-18. [CrossRef]

6. Brian A. Vanderbrink, Michael C. Ost , Ardeshir Rastinehad, Ann Anderson, Gopal H. Badlani , Arthur D. Smith , Michael A. Levine, Benjamin R. Lee . 2007. Laparoscopic Versus Open Radical Nephrectomy for Xanthogranulomatous Pyelonephritis: Contemporary Outcomes AnalysisLaparoscopic Versus Open Radical Nephrectomy for Xanthogranulomatous Pyelonephritis: Contemporary Outcomes Analysis. Journal of Endourology 21:1, 65-70. [Abstract] [PDF] [PDF Plus]

7. Pranjal Modi , Gaurang Kadam , Rajiv Goel . 2007. Retroperitoneoscopic Nephrectomy for Pyonephrotic KidneysRetroperitoneoscopic Nephrectomy for Pyonephrotic Kidneys. Journal of Endourology 21:1, 75-77. [Abstract] [PDF] [PDF Plus] 